\title{
Verletzbare Regeln in Strassenverkehr Und Syntax
}

\author{
von Gereon Müller
}

\section{Einleitung}

Grammatiken sind Regelsysteme. Üblicherweise wird angenommen, dass Regelsysteme für natürliche Sprachen mit Regeln arbeiten, die nicht verletzbar und nicht gewichtet sind; diese Standardgrammatiken gehen im Wesentlichen auf Noam Chomsky zurück. Seit Beginn der Neunzigerjahre ist jedoch mit der Optimalitätstheorie von Alan Prince, Paul Smolensky und John McCarthy ein neuer Grammatiktyp entwickelt worden, der ganz wesentlich auf verletzbaren und gewichteten Regeln beruht. ${ }^{1}$ Ziel der folgenden Ausführungen ist es, die Optimalitätstheorie zunächst auf der Basis eines weithin bekannten nichtsprachlichen Regelsystems, der Straßenverkehrsordnung ( $\mathrm{StVO}$ ), vorzustellen, und im Anschluss daran zu skizzieren, wie dieser Typ von Regelsystem für die deutsche Syntax genutzt werden kann.

\section{Straßenverkehrsordnung}

Die Urheber der StVO können von den jüngsten grammatiktheoretischen Entwicklungen in den USA noch keine Kenntnis gehabt haben; aber das von ihnen geschaffene Vorfahrtsregelsystem mit seinen verletzbaren und gewichteten Regeln ist nichtsdestoweniger bereits als optimalitätstheoretische Grammatik angelegt. Da die StVO allen Lesern vertraut ist, bietet sie sich für eine Darstellung der Grundkonzepte der Optimalitätstheorie an. Die Aufgabe der StVO (also, wenn man so will, der Grammatik des Straßenverkehrs) im Bereich der Vorfahrtsregelung ist es, für Situationen, die sich bei gleichzeitiger Ankunft von zwei oder mehr Verkehrsteilnehmern an einer Kreuzung (bzw. Einmündung) ergeben, eindeutige Strategien zur Auflösung dieser Situationen vorzugeben, also normalerweise einem Verkehrsteilnehmer die Vorfahrt zu- 


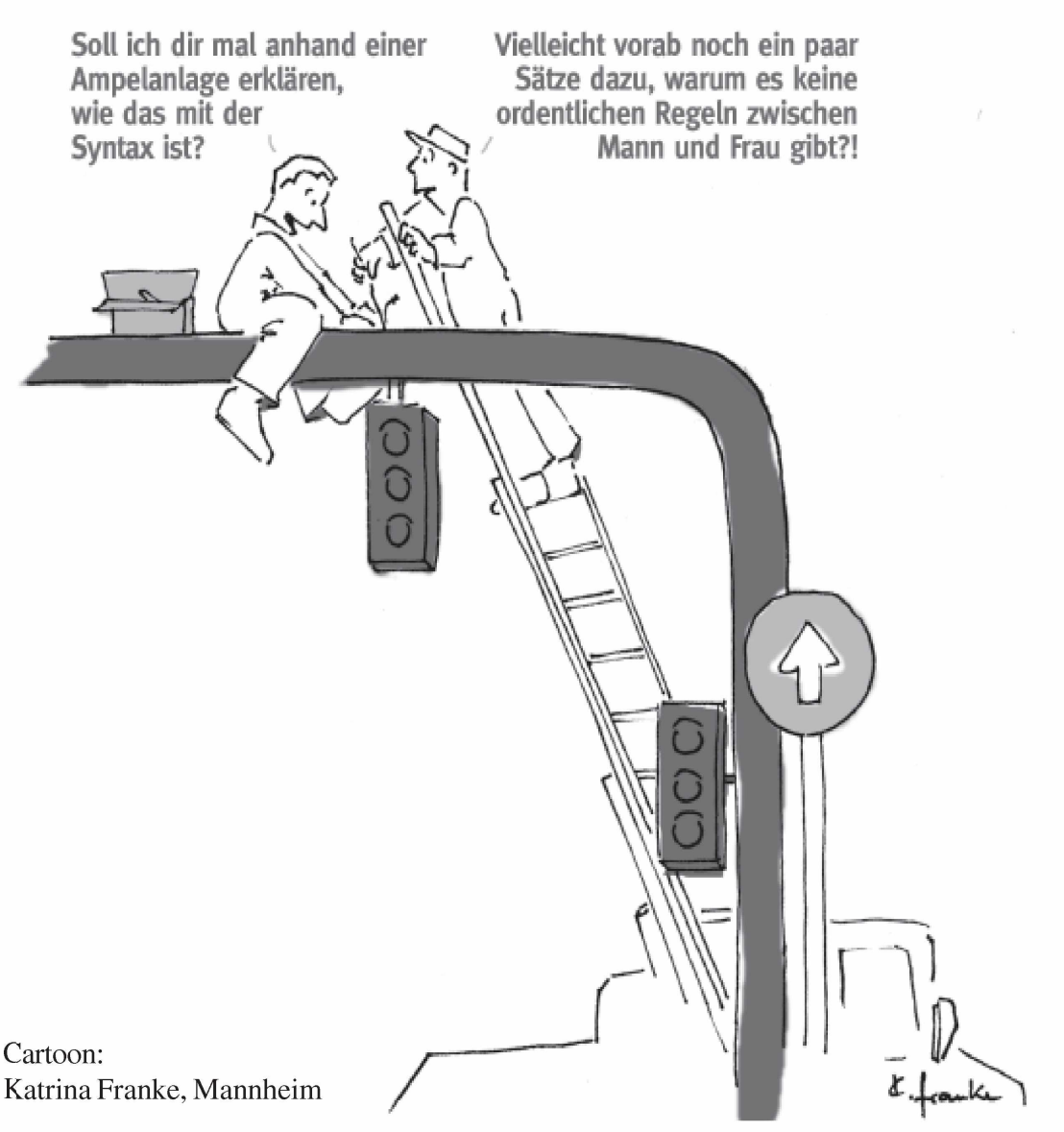

nehmer haben sofort freie Bahn zu schaffen.«>>

c. L(icht)-Zei(chen) (StVO § 37): An Kreuzungen bzw. Einmündungen bedeuten Grün: »Der Verkehr ist freigegeben «; Gelb: »Vor der Kreuzung auf das nächste Zeichen warten«; Rot: »Halt vor der Kreuzung «. >> (»Lichtzeichen gehen Vorrangregeln [und] vorrangregelnden Verkehrsschildern ... vor.«)

d. V(erkehrs)-Zei(chen)(a) (StVO § 39): Verkehrszeichen auf einem Fahrzeug ist Folge zu leisten. Sie gelten auch, wenn das Fahrzeug sich bewegt. $>>(»$ Sie gehen den Anordnungen der ortsfest angebrachten Verkehrszeichen vor.«)

e. V(erkehrs)-Zei(chen)(b) (StVO § 39): Ortsfesten Verkehrszeichen ist Folge zu leisten. $>>$ (»Regelungen durch Verkehrszeichen gehen den allgemeinen Verkehrsregeln vor.«)

f. $\mathbf{S}($ traße) v(or) F(eldweg) (StVO § 8): Fahrzeuge, die aus einem Feld- oder Waldweg auf eine andere Straße kommen, haben Vorfahrt zu gewähren. >>

g. $\mathbf{R}$ (echts) v(or) L(inks) (StVO $\S 8$ ): An Kreuzungen und Einmündungen hat die Vorfahrt, wer von rechts kommt. zuweisen. Die Ausgangssituationen können wir Inputs nennen; die zunächst einmal denkbaren Strategien zur Auflösung sind dann Outputs. Zwischen den Outputs besteht ein Wettbewerb. Derjenige Output, der aus dem Wettbewerb als Gewinner hervorgeht und somit die korrekte (»grammatische ) Strategie repräsentiert, heißt optimaler Output; alle nicht optimalen Outputs sind Verstöße gegen die StVO (somit gleichermaßen »ungrammatisch «). Wie gewinnt nun ein Output den optimalitätstheoretischen Wettbewerb? Er muss in optimaler Weise, d.h. besser als alle konkurrierenden Outputs (die auf denselben Input zurückgehen), die verletzbaren und gewichteten Vorfahrtsregeln der StVO erfüllen (genauer: im Vergleich mit jedem konkurrierenden Output bei der höchst-geordneten Regel, wo sich die beiden unterscheiden, besser abschneiden). Ein optimaler Output muss aber nicht, und dies ist entscheidend, alle Regeln respektieren.

Die deutschen Vorfahrtsregeln sind im Folgenden aufgelistet und ihrer Wichtigkeit nach geordnet. ${ }^{2}$ Als Ordnungssymbol wird das Zeichen $>>$ verwendet.

\section{Vorfahrtsregeln:}

a. V(erkehrs)-Pol(izist) (StVO § 36): Die Zeichen und Weisungen der Polizeibeamten auf der Kreuzung sind zu befolgen. $\gg(\gg$ Sie gehen allen anderen Anordnungen und sonstigen Regeln vor.«)

b. BI(aulicht)-Ein(satzhorn) (StVO § 35, 38): Fahrzeuge des Rettungsdienstes und der Polizei dürfen blaues Blinklicht zusammen mit einem Einsatzhorn verwenden; dies ordnet an: »Alle übrigen Verkehrsteil-
Diese Regeln müssen verletzbar sein, denn es kommt schnell zu Situationen, wo sie Unvereinbares verlangen. Derartige Regelkonflikte werden dann durch Regelgewichtung aufgelöst: Die höher geordnete, stärkere Regel setzt sich durch; tiefer geordnete, schwächere Regeln können verletzt werden. Als erstes Beispiel betrachte man den folgenden Input: An einer Kreuzung ohne Ampel kommt Verkehrsteilnehmer A von links auf einer Straße mit Vorfahrtsschild, B kommt von rechts auf einer Straße mit Vorfahrt-Gewähren-Schild. Ein Regelkonflikt entsteht, denn V-Zei und RvL können nicht gleichzeitig erfüllt werden: V-Zei gibt A die Vorfahrt, RvL B. Dieser Konflikt wird durch die Ordnung V-Zei $>>$ RvL aufgelöst: Der optimale, einzig korrekte Output ist der, dass A vor B die Kreuzung überqueren darf. Diese Strategie verletzt zwar die tiefer geordnete Regel RvL; diese Verletzung kann und muss aber in Kauf genommen werden, weil alle alternativen Strategien (z.B., dass B Vorfahrt erhält, oder dass beide gleichzeitig fahren) die höher geordnete Regel V-Zei verletzen müssen. Optimalitätstheoretische Wettbewerbe lassen sich mit Hilfe von Tabellen darstellen. Den soeben skizzierten Wettbewerb illustriert Tabelle T1.

T1: V-Zei $\gg$ RvL
\begin{tabular}{|l|c|c|c|c|c|c|}
\hline $\begin{array}{l}\text { I: A(L, } \\
\text { B(R, })\end{array}$ & V-Pol & Bl-Ein & Li-Zei & V-Zei & SvF & RvL \\
\hline O1: A vor B & & & & & & $*$ \\
\hline O2: B vor A & & & & $* !$ & & \\
\hline O3: A und B & & & & $* !$ & & $*$ \\
\hline
\end{tabular}

Hier entspricht die Reihenfolge der Vorfahrtsregeln von links nach rechts ihrer relativen Gewichtung. Die 
Optimalität eines Outputs (hier: von O1) wird durch eine zeigende Hand $(+)$ signalisiert. Eine Verletzung einer Regel wird in der Tabelle als Stern (*) eingetragen; ist eine Regelverletzung fatal für einen Output-Kandidaten $\mathrm{O}$ (also für seine Nicht-Optimalität verantwortlich), wird dem Stern ein Ausrufezeichen (*!) beigefügt. Die Optimalität eines Outputs lässt sich in Tabellen immer leicht ermitteln: Es ist derjenige Output optimal, der den ersten Stern am weitesten rechts in der Tabelle hat. Diese Notationskonventionen sind Standard. Zusätzlich gibt es in T1 aber auch noch einige Abkürzungen, die ich auf die spezielle Anwendung des Modells hin gewählt habe: Der Input I, der die Ausgangssituation beschreibt, wird hier und im Folgenden für die Verkehrsteilnehmer mit Hilfe von Merkmalen wie L (»kommt von links«), R (»kommt von rechts «), u (»hat ein Vorfahrtsschild «) und $t$ (»hat ein Vorfahrt-Gewähren-Schild «) erfasst.

Die Ausgangssituation in T1 kann nun minimal modifiziert werden, etwa dergestalt, dass zusätzlich noch eine Ampel an der Kreuzung steht, die für A rot ist und für B grün. (Ampeln an Kreuzungen, an denen auch Verkehrsschilder stehen, sind keine Seltenheit.) Nun kommt es zum Konflikt der Regeln Li-Zei und V-Zei, und die oben angeführte Ordnung stellt sicher, dass V-Zei im Zweifelsfall verletzt werden kann und B vor A die Kreuzung überqueren muss. Dies zeigt Tabelle T2. (Dass in T2 vom optimalen Output $\mathrm{O} 2$ auch wiederum die tiefstgeordnete Regel RvL erfüllt wird, ist ein Nebeneffekt, der jedoch im Prinzip irrelevant ist - man kann sich leicht klar machen, dass $\mathrm{O} 2$ auch optimal wäre, wenn im Input B nicht von rechts, sondern von links käme.)

\begin{tabular}{|c|c|c|c|c|c|c|}
\hline 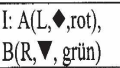 & V-Pol & Bl-Ein & Li-Zei & V-Zei & $\mathrm{SvF}$ & RvL \\
\hline $01: A$ vor B & & & *! & & & * \\
\hline $02: B$ vor $A$ & & & & $*$ & & \\
\hline $03: A$ und $B$ & & & $* !$ & * & & * \\
\hline
\end{tabular}

Wir können die Ausgangslage noch etwas komplizierter machen. Angenommen, alles ist so wie im Input von T2, mit der einzigen Ausnahme, dass A ein Krankenwagen ist, der Blaulicht und Martinshorn eingeschaltet hat. Nun kommt es zum Konflikt zwischen Bl-Ein und Li-Zei. Die Auflösung des Konflikts zeigt Tabelle T3: A erhält unter Verletzung von Li-Zei (und RvL) die Vorfahrt (X soll hier für blaues Blinklicht und Einsatzhorn stehen.)

\begin{tabular}{|c|c|c|c|c|c|c|}
\hline $\begin{array}{l}\text { I: A(L, rot, **), } \\
\text { B(R, }, \text { grün) }\end{array}$ & V-Pol & Bl-Ein & Li-Zei & V-Zei & SvF & $\mathrm{RvL}$ \\
\hline $01: A$ vor $B$ & & & $*$ & & & $*$ \\
\hline $02: B$ vor $A$ & & $* !$ & & $*$ & & \\
\hline $03: A$ und $B$ & & *! & $*$ & $*$ & & $*$ \\
\hline
\end{tabular}

Bislang ist die höchst-geordnete Regel V-Pol noch nicht relevant gewesen. Es sei nun angenommen, dass A hinsichtlich der tiefer geordneten Regeln zunächst einmal wesentlich schlechter als B abschneidet: A kommt von links, ein Verkehrszeichen gebietet Halt, und die Ampel ist rot. Soweit gibt es keinen Regelkonflikt, und wir erwarten Vorfahrt für B. Allerdings steht nun auch noch ein Verkehrs- polizist auf der Kreuzung, und der signalisiert freie Fahrt für A und Halt für B. Damit entsteht ein Regelkonflikt, und die optimale und somit einzig korrekte Auflösung ist, dass A vor B die Kreuzung überquert; vgl. Tabelle T4 (wobei : für ein durch den Verkehrspolizisten erteiltes Haltegebot und ùfür die Fahrtfreigabe steht).

T4: V-Pol $\gg$ andere Regeln

\begin{tabular}{|c|c|c|c|c|c|c|}
\hline 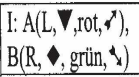 & V-Pol & Bl-Ein & Li-Zei & V-Zei & $\mathrm{SvF}$ & $\mathrm{RvL}$ \\
\hline $01: A$ vor $B$ & & & $*$ & * & & * \\
\hline O2: B vor A & $* !$ & & & & & \\
\hline $03: A$ und $B$ & $* !$ & & * & * & & * \\
\hline
\end{tabular}

Zugegebenermaßen ist die Ausgangssituation keine, mit der man alle Tage konfrontiert wird. Sie ist aber durchaus möglich (z.B., wenn die Ampelanlage in Betrieb ist, aber nicht mehr von Rot auf Grün bzw. Grün auf Rot wechselt und gerade auch nicht ausgeschaltet werden kann oder soll), und der entscheidende Punkt ist, dass die StVO eindeutig regelt, wie in einer solchen Situation zu verfahren ist. Grundsätzlich würde sich am Resultat auch nichts ändern, wenn B in T4 noch dazu ein Krankenwagen mit Blaulicht wäre. Man könnte sich dann zwar fragen, warum der Vekehrspolizist B nicht sofort Vorfahrt einräumt; daran, dass A Vorfahrt hat, wenn nur so V-Pol erfüllt wird, gibt es aber nichts zu rütteln. Die außergewöhnliche Situation in T4 habe ich deshalb gewählt, weil sich damit besonders gut ein allgemeines optimalitätsgrammatisches Gesetz illustrieren lässt: Qualität geht vor Quantität; keine noch so große Zahl von Verletzungen tiefer geordneter Regeln wiegt auch nur eine einzige Verletzung einer höher geordneten Regel auf.

Ein gefährlicher Konkurrent muss in den bisherigen Wettbewerben noch als nicht optimal ausgeschlossen werden, und zwar der, der den Input überhaupt nicht als Output mit Handlungsanweisung realisiert, sondern als Unterlassung einer Handlung. Ein solcher leerer Output Ø (ein sog. NullParse) zeigt zwar ein an sich hervorragendes Verhalten gegenüber den Regeln (wo kein Verkehrsteilnehmer losfährt, wird auch keine Vorfahrtsregel missachtet); wir müssen aber sicherstellen, dass er nicht allzu oft optimal werden kann, denn dann würde natürlich kein Verkehr mehr fließen können. Der leere Output $\varnothing$ verletzt glücklicherweise eine hoch geordnete (wenn auch eher implizit vorausgesetze) Regel V(erkehrs)-Real(isierung), derzufolge jede Ausgangssituation im Straßenverkehr eine nicht-triviale klare Auflösung erhalten muss, d.h.: Ein Output führt auf eindeutige Weise zu fließendem Verkehr.

Wenn V-Real in der StVO über Bl-Ein geordnet ist, ist gewährleistet, dass die bislang diskutierten Ausgangssituationen jeweils wie angegeben aufgelöst werden und der Verkehr nicht zum Erliegen kommt. Eine interessante Konsequenz ergibt sich jedoch, wenn wir annehmen, dass die hoch geordnete Regel V-Real ihrerseits nicht ganz undominiert ist, sondern immerhin noch unterhalb von $\mathbf{V}$ Pol angeordnet ist. Denn dann folgt, dass der leere Output O4 - wie in T5 gezeigt - optimal werden kann, wenn ein Verkehrspolizist - aus welchem Grund auch immer - A 
und B gleichermaßen Halt anordnet, und zwar ganz unabhängig davon, wie sich A und B hinsichtlich der tiefer geordneten Regeln verhalten. (Angenommen ist ansonsten derselbe Input wie in T2.)

\begin{tabular}{|c|c|c|c|c|c|c|}
\hline 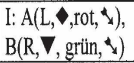 & V-Pol & V-Real & Li-Zei & V-Zei & SvF & RvL \\
\hline $01: A$ vor $B$ & $* !$ & & * & & & * \\
\hline $02: \mathrm{B}$ vor $\mathrm{A}$ & *! & & & * & & \\
\hline 03: $A$ und $B$ & *! & $*$ & $*$ & $*$ & & * \\
\hline $04: \varnothing$ & & $*$ & & & & \\
\hline
\end{tabular}

Aufgrund der Forderung nach eindeutiger Verkehrsauflösung bestraft V-Real nicht nur den leeren Output $\varnothing$, sondern auch immer noch Outputs wie O3 in T5, bei denen mehr als ein Verkehrsteilnehmer losfährt; und dies ist natürlich auch nur gut so. (So, wie das System angelegt ist, könnte bei gleichzeitiger Fahrtfreigabe für A und B durch einen Verkehrspolizisten auch O3 optimal werden. Tatsächlich tritt dieser Fall unter gewöhnlichen Umständen wohl aber nur als Versehen ein (zumal die konventionalisierte Körperhaltung des Verkehrspolizisten ein solches Gebot erschwert); und dann wird man als Verkehrsteilnehmer bemüht sein, sich über den optimalen Output hinwegzusetzen und eine »ungrammatische« Lösung zu verfolgen; ganz allgemein wird man nicht unbedingt die von den gewichteten Verkehrsregeln vorgegebene Auflösung einer Verkehrssituation wählen, wenn das zu einem Unfall o. Ä. führt. Es manifestiert sich hier schlicht das Phänomen, dass man sich nicht notwendigerweise an das halten muss, was ein Regelsystem vorschreibt, und dass eine dem System zuwiderlaufende Strategie manchmal aus system-externen Gründen vorzuziehen ist.)

Das große Gewicht von V-Real kann in ein Dilemma führen. Angenommen, vier Verkehrsteilnehmer A, B, C, D kommen gleichzeitig an eine Straßenkreuzung ohne Verkehrszeichen, Ampelanlage oder andere Gegebenheiten, die eine höher geordnete Regel aktivieren. Dann verletzt zunächst einmal jeder Output V-Real, und zwar entweder, weil keiner fährt, oder weil mehr als einer fährt, oder weil, wenn z.B. A (unter Verletzung der tief geordneten Regel RvL) allein fährt, dies nicht auf eindeutige Weise zu flieBendem Verkehr führt, denn wenn B oder C oder D fahren, wird dies ja als gleich gut klassifiziert. ${ }^{3}$ Für dieses Dilemma lernt man in der Fahrschule einen besonderen Typ der Auflösung: Hier, und nur hier, kann als Reparatur, als letzter Ausweg, angesichts eines ansonsten zu Inakzeptabilität führenden Inputs eine hoch geordnete (aber unter V-Real stehende) Regel A(bsprache)-Ver(bot) verletzt werden, derzufolge Vorfahrtsregelung nicht per Absprache erfolgen darf: Die Verkehrsteilnehmer einigen sich, wer zuerst fährt. Die danach folgenden Optimierungsschritte werden dann wieder ganz klassisch gemäß RvL durchgeführt. Generell gilt, dass in »normalen « Verkehrssituationen derartige AVer verletzende Reparaturmaßnahmen ausgeschlossen sind: Wenn es nicht die einzige Möglichkeit ist, V-Real zu erfüllen, ist eine Verletzung von A-Ver fatal.

Fassen wir zusammen: Die StVO ist als optimalitätstheoretisches Regelsystem zu betrachten, weil es bei den Vorfahrtsregeln zu Regelkonflikten kommt. Diese Kon- flikte werden durch Regelgewichtung aufgelöst, und zwar so, dass im Zweifelsfall die Qualität von Regelverletzungen entscheidend ist, nicht die Quantität. ${ }^{4}$ Als Nebeneffekt lässt sich das Konzept der Reparatur (des letzten Auswegs) gut erfassen: Wenn alle Alternativen noch schlechter sind, kann wie bei der Diskussion des Abspracheverbots gesehen ein Output, der normalerweise nicht vom Regelsystem toleriert wird, doch optimal werden. In Systemen mit unverletzbaren und nicht gewichteten Regeln ist für ein solches Reparaturkonzept kein Platz. Schließlich ermöglicht eine optimalitätstheoretische Herangehensweise, das Konzept des unmarkierten Falles (Normalfalles) zu erfassen, das in der Sprachwissenschaft von einiger Bedeutung ist (s.u.): Wenn nicht speziell etwas Gegenteiliges verlangt wird, tritt in der StVO die tiefstgeordnete Regel RvL hervor; oder, in linguistischer Redeweise: Im unmarkierten Fall gilt in der StVO rechts vor links. Auch für dieses Konzept gibt es in Systemen mit unverletzbaren und nicht gewichteten Regeln kaum Raum. Regelkonflikt, Reparatur und unmarkierter Fall sind nun auch in der deutschen Syntax ganz wichtige Konzepte. Der folgende Abschnitt soll zeigen, dass ein optimalitätstheoretischer Ansatz auch in diesem Bereich gut motiviert ist.

\section{Syntax}

Die Optimalitätstheorie funktioniert in der Syntax im Prinzip genauso wie im Straßenverkehr. Wir müssen natürlich etwas andere Annahmen über die Natur von Input und Output machen. Angenommen sei, dass im syntaktischen Input die bedeutungstragenden Wörter versammelt sind, die im Satz verwendet werden sollen, und dass darüber hinaus die Bedeutung, die der Satz haben soll, vorgegeben ist. Auf der Basis des Inputs erzeugt zunächst eine primitive (Vor-)Grammatik, der sog. Generator, mögliche Sätze als Outputs. (Im Straßenverkehr gibt es einen solchen Outputgenerator zwar im Prinzip auch; seine Aufgabe ist jedoch trivial.) Die Zahl der konkurrierenden Outputs für einen gegebenen Input ist in der Syntax aufgrund der hier verfügbaren rekursiven Operationen sehr hoch und potenziell nicht endlich. In den Tabellen beschränkt man sich aber normalerweise auf einige wenige Kandidaten, nämlich die Outputs, die am ehesten eine Chance haben, optimal zu werden. Gemäß den verletzbaren und gewichteten Regeln wird dann aus den vom Generator erzeugten konkurrierenden Outputs der optimale, einzig grammatische Satz ermittelt.

\section{Regelkonflikt}

Beginnen wir mit den Regelkonflikten. Dass im Bereich der deutschen Syntax fast unvermeidlich Konflikte zwischen unabhängig begründeten Regeln entstehen, ist schon oft festgestellt worden. Ein einfaches Beispiel betrifft die Fragesatzbildung. Einerseits muss ein W-Element (also ein Fragewort wie wem oder eine Phrase mit einem Fragewort wie welchem Mann) in Ergänzungsfragen am Satzanfang stehen; wenn wir die klassische Felderlehre mit 
Vorfeld, Mittelfeld und Nachfeld zu Grunde legen, können wir (zumindest für die gegenwärtigen Zwecke) sagen: im Vorfeld. Dies ist die F(ragesatz)-Regel. Andererseits ist auch eine potenziell konfligierende Regel gut bestätigt, dass nämlich Komplemente des Verbs im Mittelfeld zu stehen haben. Das ist die M(ittelfeld)-Regel. In Fragesätzen kommt es somit im Deutschen oft zum Regelkonflikt. Die empirische Evidenz sagt uns, dass die F-Regel höher geordnet und die M-Regel somit zugunsten der F-Regel verletzbar ist, denn es heißt Ich frage mich, wem er das Buch gegeben hat und nicht *Ich frage mich, dass er wem das Buch gegeben hat. Diesen Wettbewerb illustriert Tabelle T6.

T6: F-Regel $\gg$ M-Regel

\begin{tabular}{|l|c|c|}
\hline I: er, wem, ... & F-Regel & M-Regel \\
\hline $01 ; \ldots$ wem er das Buch gegeben hat & & $*$ \\
\hline $02: \ldots$ dass er wem das Buch gegeben hat & $* !$ & \\
\hline
\end{tabular}

Was kann man angesichts dieser Evidenz in Standardgrammatiken tun, die nur nicht verletzbare und nicht gewichtete Regeln kennen? Man muss wohl entweder sagen, dass die M-Regel nicht gelten darf; oder man muss die MRegel ad hoc um eine Ausnahmeklausel bereichern (»gilt nicht für W-Elemente «); oder, und das ist ein beliebter Ausweg in der chomskyschen Tradition, man muss stipulieren, dass beide Regeln gelten, aber nicht auf derselben Ebene (die F-Regel gilt dann z.B. auf der Oberflächenstruktur, die M-Regel auf einer abstrakten Tiefenstruktur). Im Lichte dieser Alternativen erscheint die optimalitätstheoretische Herangehensweise eleganter, umso mehr, als sich zeigt, dass die F-Regel ihrerseits auch nicht unverletzbar ist. Betrachten wir den Fall, dass in einem Satz zwei WElemente auftreten, also z.B. wem und welches Buch, und eine Mehrfachfrage bilden. Dann fordert die F-Regel, dass beide W-Elemente im Vorfeld stehen. In manchen Sprachen (z.B. dem Bulgarischen) tritt auch genau dies ein; im Deutschen jedoch nicht: Es heißt Ich weiß nicht, wem er welches Buch gegeben hat und nicht *Ich weiß nicht, wem welches Buch er gegeben hat. In Standardgrammatiken muss man nun die F-Regel aufgeben oder verkomplizieren. In einer optimalitätstheoretischen Grammatik ergibt sich die Beobachtung zwanglos aus dem großen Gewicht einer weiteren Regel, derzufolge im Vorfeld höchstens ein Element stehen darf. Diese V(orfeld)-Regel muss man ohnehin annehmen, um die Ungrammatikalität von Sätzen wie *Der Fritz das Buch hat gestern gekauft zu erfassen. Bei einer Ordnung V-Regel $>>$ F-Regel $>>$ M-Regel folgt, dass in Fragesätzen die F-Regel unter Verletzung der MRegel erfüllt wird, aber nur soweit, wie es die höher geordnete V-Regel zulässt. Dies veranschaulicht Tabelle T7.

T7: V-Regel $\gg$ F-Regel

\begin{tabular}{|c|c|c|c|}
\hline I: er, wem, welches, ... & V-Regel & F-Regel & M-Regel \\
\hline 01:... dass er wem welches Buch gegeben hat & & $* * !$ & \\
\hline -02:... wem er welches Buch gegeben hat & & ${ }^{*}$ & ${ }^{*}$ \\
\hline 03:... wem welches Buch er gegeben hat & $* !$ & & ${ }^{* *}$ \\
\hline
\end{tabular}

Ein zweiter Bereich neben der Fragesatzbildung, an dem sich Regelkonflikte in der deutschen Syntax gut illustrieren lassen, betrifft die Wortstellung im Mittelfeld. Wie häufig festgestellt worden ist, ergibt sich die Abfolge der
Nominalphrasen (NPs) im deutschen Mittelfeld aus der Interaktion sehr allgemeiner, aber konfligierender und relativ zueinander gewichteter Regeln. ${ }^{5}$ Diese Beobachtung ist in Standardgrammatiken nur schwer integrierbar; sie fügt sich jedoch unmittelbar in einen optimalitätstheoretischen Ansatz ein. Drei Wortstellungsregeln seien betrachtet: Die Pron(omen)-Regel besagt, dass im Mittelfeld pronominale NPs vor nicht-pronominalen NPs stehen; die Bel(ebtheits)-Regel linearisiert belebte NPs vor unbelebten NPs; und die Dat(iv)-Regel fordert, dass Dativ-NPs vor Akkusativ-NPs kommen. Jede dieser Regeln ist an sich gut begründet; aber zusammen können sie von Sätzen sehr schnell Unvereinbares verlangen. Wenn Konflikte entstehen, werden sie auf der Basis der relativen Gewichtung Pron-Regel $>>$ Bel-Regel $>>$ Dat-Regel aufgelöst.

Angenommen sei z.B., dass im Input bei einem Verb mit zwei Objekten das eine ein belebtes Akkusativobjekt ist, das andere ein unbelebtes Dativobjekt. Dann kommt es zum Konflikt von Bel-Regel und Dat-Regel, und da erstere Regel per Annahme die höher geordnete ist, wird korrekt erfasst, dass es heißt Maria hat die Kinder dem Einfluss entzogen und nicht??Maria hat dem Einfluss die Kinder entzogen, d.h., dass hier das Akkusativobjekt dem Dativobjekt vorangeht; vgl. Tabelle T8.

T8: Bel-Regel $\gg$ Dat-Regel

\begin{tabular}{|l|c|c|}
\hline I: Kinder, Einfluss, die, dem, entzogen, ... & Bel-Regel & Dat-Regel \\
\hline 01:... Maria hat die Kinder dem Einfluss entzogen & & $*$ \\
\hline 02: ... Maria hat dem Einfluss die Kinder entzogen & $* !$ & \\
\hline
\end{tabular}

Vorausgesagt ist somit, dass bei identischem Belebtheitsstatus der beiden Objekte beim selben Verb die tief geordnete Dat-Regel relevant werden und die Abfolge determinieren kann. Wie der Kontrast von ??Maria hat die Kinder dem Fritz entzogen gegenüber Maria hat dem Fritz die Kinder entzogen zeigt, ist genau dies der Fall; vgl. Tabelle T9.

T9: Dat-Regel

\begin{tabular}{|c|c|c|}
\hline I: Kinder, Fritz, die, dem, entzogen, ... & Bel-Regel & Dat-Regel \\
\hline 01: Maria hat die Kinder dem Fritz entzogen & & $* !$ \\
\hline 02: Maria hat dem Fritz die Kinder entzogen & & \\
\hline
\end{tabular}

Die Bel-Regel ist zwar oft, aber längst nicht immer entscheidend für die Abfolge von Objekten im Mittelfeld: Eine höher geordnete Regel kann ihre Effekte außer Kraft setzen. Liegen im Input z.B. ein belebtes Dativobjekt und ein unbelebtes Akkusativobjekt vor (was die prototypische Situation bei ditransitiven Verben ist), dann favorisieren Bel-Regel und Dat-Regel zwar dieselbe Abfolge; ist jedoch das Akkusativobjekt ein Pronomen, setzt die PronRegel wie in Tabelle T10 gezeigt die umgekehrte Abfolge durch, unter Verletzung der tiefer geordneten Regeln; vgl. Maria hat es dem Fritz entzogen gegenüber * Maria hat dem Fritz es entzogen (wobei das Pronomen für eine unbelebte Entität stehen soll, z.B. für ein Projekt).

T10: Pron-Regel $\gg$ Bel-Regel

\begin{tabular}{|c|c|c|c|}
\hline I: es, Fritz, dem, entzogen, ... & Pron-Regel & Bel-Regel & Dat-Regel \\
\hline 01: Maria hat es dem Fritz entzogen & & $*$ & $*$ \\
\hline 02: Maria hat dem Fritz es entzogen & $* !$ & & \\
\hline
\end{tabular}


Diese Daten zeigen übrigens gut, dass es im Deutschen keine feste Grundabfolge der NP-Komplemente geben kann, weder allgemein noch verbspezifisch. Vielmehr ist die Grundabfolge variabel und jeweils Resultat der Optmierung. Bevor ich nun zum nächsten Punkt (Reparatur) komme, sei noch auf eine Komplikation hingewiesen, die sich aus den soeben betrachteten Wortstellungsdaten ergibt: Ein Grundsatz der Optimalitätstheorie ist, dass nicht-optimale Sätze alle gleichermaßen ungrammatisch sind. Dies ist zwar für viele Bereiche (z.B. die oben diskutierten nicht-optimalen Fragesatzvarianten) auch erwünscht; für die Abfolge im deutschen Mittelfeld lässt sich eine solch scharfe Vorhersage aber vielleicht nicht immer aufrechterhalten. Hier sind dann Zusatzannahmen notwendig.

\section{Reparatur}

Ein klassisches Reparaturphänomen in der deutschen Syntax ist der nicht umsonst so genannte Ersatzinfinitiv. Dabei handelt es sich um die Erscheinung, dass, wenn z.B. ein Modalverb wie wollen unter dem Hilfsverb haben eingebettet ist und selbst wiederum einen Infinitiv einbettet, das Modalverb anstatt in der eigentlich zu erwartenden Partizipform im zunächst einmal überraschenden Infinitiv auftritt. Das heißt, während normalerweise der korrekten Form dass sie das gewollt hat die ungrammatische Variante *dass sie das hat wollen gegenübersteht, wird der Ersatzinfinitv bei weiterer Infinitiveinbettung durch das Modalverb die einzige Möglichkeit: Anstelle des erwarteten *dass sie das Lied singen gewollt hat erscheint dass sie das Lied hat singen wollen. Die Wahl der Ersatzinfinitivform ist eine Reparatur, die nur möglich ist, wenn ansonsten eine wichtigere Regel verletzt würde. Dies lässt sich optimalitätstheoretisch auf der Basis der folgenden zwei Regeln erfassen: ${ }^{6}$ Die Part(izip)-Regel setzt fest, dass Modalpartizipien selbst keine verbalen Satzglieder einbetten dürfen; und eine allgemeine Sel(ektions)-Regel verlangt, dass lexikalisch im Input vermerkte Selektionseigenschaften in der Syntax nicht geändert werden dürfen. (Diese Regel verlangt also »Treue« zum Input; der englische Fachterminus für diesen Regeltyp ist dementsprechend »faithfulness constraint.«) Konkret besagt die SelRegel für den vorliegenden Fall: Ein lexikalisch selegiertes Partizip Perfekt darf nicht zum bloßen Infinitiv werden. Bei einer Gewichtung Part-Regel $\gg$ Sel-Regel ergibt sich die Optimalität des Ersatzinfinitivs wie in Tabelle T11 gezeigt.

T11: Part-Regel $>>$ Sel-Regel

\begin{tabular}{|c|c|c|}
\hline $\mathrm{I}:$ hat, gewollt, singen, ... & Part-Regel & Sel-Regel \\
\hline $01: \ldots$ singen gewollt hat & $* !$ & \\
\hline $02: . .$. hat singen wollen & & $*$ \\
\hline
\end{tabular}

Ist jedoch die Part-Regel auch ohne Treueverletzung erfüllbar (weil das Modalverb kein weiteres verbales Element einbettet), wird die tiefer geordnete Sel-Regel entscheidend und blockiert erfolgreich den Ersatzinfinitiv; vgl. Tabelle T12.
T12: Sel-Regel

\begin{tabular}{|l|c|c|}
\hline I: hat, gewollt, ... & Part-Regel & Sel-Regel \\
\hline 01:... das gewollt hat & & \\
\hline $02: \ldots$ hat das wollen & & $* !$ \\
\hline
\end{tabular}

Auf diese Weise lässt sich in der Optimalitätstheorie gut die klassische Sicht auf den Ersatzinfinitiv nachzeichnen, die sich im Namen des Phänomens dokumentiert. Eine umfassende Analyse hat allerdings noch weitere Gesichtspunkte zu berücksichtigen, die hier ausgeblendet sind: Zum einen geht mit dem Formwechsel beim Ersatzinfinitiv auch ein Wortstellungswechsel einher (dies kann man so interpretieren, dass Verletzungen der Sel-Regel in einer Position hinter dem Finitum weniger schwer wiegen als vor dem Finitum). Zum anderen ist die Part-Regel in der oben angeführten Form nicht allgemein genug; sie wäre weiter aufzugliedern, so dass die Optionalität des Ersatzinfinitivs bei Perzeptionsverben wie hören und das Verbot des Ersatzinfinitivs bei Kontrollverben wie versuchen erfasst werden kann. Ungeachtet derartiger Komplikationen bleibt jedoch festzuhalten, dass eine Standardgrammatik in diesem Bereich an ihre Grenzen stößt; denn dass der Ersatzinfinitiv mit einer Regelverletzung einher geht, lässt sich kaum überzeugend leugnen.

Beim Pronominaladverb handelt es sich im Grunde um ein ganz ähnliches Reparaturphänomen. Ein in einer Präpositionalphrase (PP) erwartetes Personalpronomen erscheint zwar auch tatsächlich, wenn das Pronomen als belebt zu verstehen ist. Wird das Pronomen jedoch unbelebt interpretiert, tritt optional ein zunächst einmal unerwartetes sog. »R-Pronomen« da auf, das mit der Präposition zusammen ein Pronominaladverb bildet (R-Pronomen deshalb, weil bei vokalischem Anlaut der Präposition ein synchron epenthetisches $r$ eingeschoben wird). Dieses RPronomen ist sogar obligatorisch, wenn es sich beim Personalpronomen um es handelt. So sagt man im Standarddeutschen zwar mit ihr zufrieden, nicht *damit zufrieden, wenn es z.B. um Maria geht; handelt es sich jedoch beispielsweise um eine Ausstellung, stehen mir ihr zufrieden und damit zufrieden nur stilistisch unterschieden nebeneinander; und es heißt ganz systematisch nicht * für es gestimmt, sondern dafür gestimmt. ${ }^{7}$ Da das R-Pronomen da im Ursprung (und in allen anderen Kontexten, in denen es auftaucht) adverbial und nicht nominal ist, liegt es nahe, Pronominaladverbien als Reparaturformen anzusehen, zu denen nur als letzte Möglichkeit Zuflucht genommen werden kann.

Eine einfache Analyse kann nun wie folgt aussehen: Es gibt eine P/Pron-Regel, derzufolge ein unbelebtes Personalpronomen (außer es) nicht von $\mathbf{P}$ regiert werden darf, und eine analoge $\mathbf{P} / \mathbf{e s}-\mathbf{R e g e l}$, derzufolge es nicht von $\mathbf{P}$ regiert werden darf. ${ }^{8}$ Dazu kommt wiederum die Sel-Regel, die für die vorliegenden Phänomene die Konsequenz hat, dass ein von P selegiertes Personalpronomen nicht durch da ersetzt werden darf. Es sei nun die P/es-Regel höher geordnet als die Sel-Regel; dann erfolgt unter Verletzung der Sel-Regel im optimalen Output innerhalb einer PP (und nur hier) obligatorisch die Ersetzung von es durch da; vgl. 


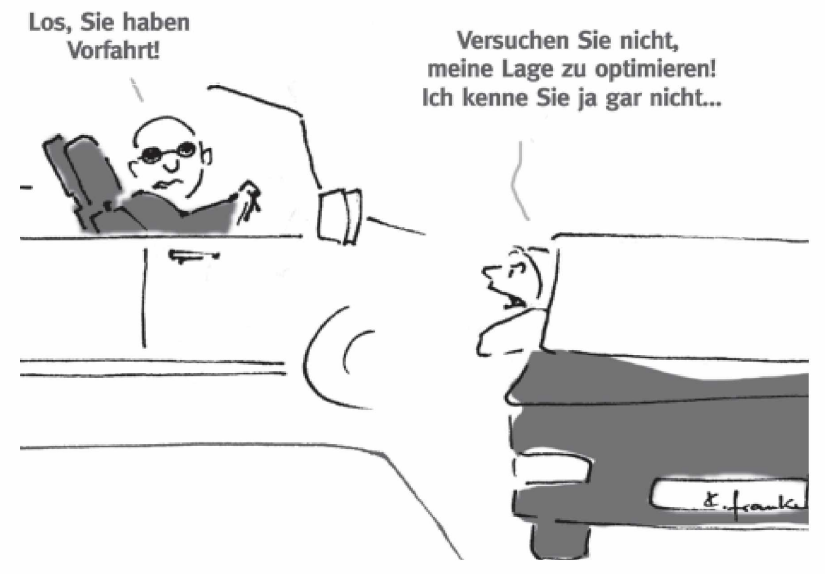

Cartoon: Katrina Franke, Mannheim

Tabelle T13. Für die Optionalität der Pronominaladverbbildung bei den anderen unbelebt interpretierten Pronomina kann man nun annehmen, dass P/Pron-Regel und Sel-Regel gleich wichtig, d.h. gekoppelt (»tied «) sind. (Dies lässt sich mit Hilfe des Symbols $\bigcirc$ sowie fehlender statt durchgezogener Linien in der Tabelle darstellen.) Dann erweisen sich die beiden Strategien als gleichermaßen optional; vgl. Tabelle T14. Nichts motiviert jedoch eine Verletzung der Sel-Regel im Falle der PP-internen belebt interpretierten Personalpronomina; daher bleibt Pronominaladverbbildung hier unmöglich. (In T14 würde in diesem Fall bei O1 unter P/Pron-Regel der Stern fehlen; die Verletzung der Sel-Regel durch O2 wird somit fatal.)

T13: P/es-Regel 》 Sel-Regel

\begin{tabular}{|l|c|c|}
\hline I: für, es, ... & P/es-Regel & Sel-Regel P/Pron-Regel \\
\hline 01:... für es gestimmt & $* !$ & \\
\hline * O2:... dafür gestimmt & & $*$ \\
\hline
\end{tabular}

T14: P/Pron-Regel O Sel-Regel

\begin{tabular}{|l|c|cc|}
\hline I: mit, , hrr(unbelebt), ... & P/es-Regel & Sel-Regel & P/Pron-Regel \\
\hline 01:... mit hihr zufrieden & & & $*$ \\
\hline 02: ... damit zuffieden & & $*$ & \\
\hline
\end{tabular}

Wiederum ist die Analyse noch zu verfeinern. Insbesondere geht wie beim Ersatzinfinitiv auch beim Pronominaladverb mit dem Formwechsel ein Wortstellungswechsel einher; interessanterweise erfolgt der jedoch gerade in die andere Richtung (das R-Pronomen erscheint links von der Präposition). Man mag spekulieren, dass die geänderte Abfolge mit der typischen Rektionsrichtung korreliert (Verben regieren im Deutschen nach links, Präpositionen nach rechts). Ich möchte das Thema hier aber nicht weiter verfolgen und mich nun dem dritten oben erwähnten Konzept zuwenden, für das man die Optimalitätstheorie gut gebrauchen kann, nämlich dem des unmarkierten Falls.

\section{Unmarkierter Fall}

Eine bestimmte Eigenschaft einer Form gilt als unmarkierter Fall, wenn sie immer dann auftritt, wenn nicht speziell Gegenteiliges verlangt wird. In Standardgrammatiken ist die theoretische Erfassung dieses Konzepts nicht unproblematisch. In einer Optimalitätsgrammatik zeigt das Vorliegen eines unmarkierten Falles die Aktivierung einer tiefer geordneten Regel, die zwar immer da ist, aber normalerweise von höher geordneten, konfligierenden Regeln überlagert wird. Wir haben bereits gesehen, dass in der StVO die tief geordnete Regel RvL den unmarkierten Fall erfasst. Analoges gilt in der Syntax für die Dat-Regel (im unmarkierten Fall geht im Mittelfeld des deutschen Satzes der Dativ dem Akkusativ voran), die Sel-Regel (im Normalfall wird lexikalische Selektion in der Syntax respektiert) und letztlich auch die M-Regel (im unmarkierten Fall steht ein Komplement im Mittelfeld). Ein klassischer Normalfall in der Syntax sei nun noch angesprochen: der des unmarkierten Kasus.

Für das Deutsche gilt, dass, wenn nicht explizit Gegenteiliges gefordert wird, eine NP den Nominativ trägt; dies ist der unmarkierte (oder »Default «-) Kasus. Der Default-Nominativ tritt zutage unter anderem in als-Konstruktionen ohne Kasuskongruenz: die Ehrung des Kanzlers als großer Politiker/*großen Politiker; in Infinitivkonstruktionen mit Bezug auf das logische Subjekt des Infinitivs: Wir baten die Männer, einer nach dem anderen/*einen nach dem anderen durch die Sperre zu gehen; bei Linksversetzung ohne Kasuskongruenz: Der Kaiser/*Den Kaiser, dem verdanken wir nichts; sowie in prädikativen undKonstruktionen: Der/*Den und ein Buch lesen? (Dass ich nicht lache!). In anderen (wenn man so will, typischeren) Kontexten, in denen eindeutig eine Kasusrektion vorgegeben ist, ist der Default-Nominativ dagegen blockiert; vgl. *dass ich er getroffen habe, *dass man der Mann gedachte oder auch *dass wir der Mann das Buch lesen sehen. Eine optimalitätstheoretische Analyse kann diesem Umstand gut dadurch Rechnung tragen, dass zu Kasusregeln wie der Gen(itiv)-Regel (das Objekt eines lexikalisch als den Genitiv regierend markierten Verbs trägt den Genitiv), der Akk(usativ)-Regel (das Objekt eines transitiven Verbs trägt den Akkusativ) und der Nom(inativ)Regel (das Subjekt eines finiten Satzes trägt den Nominativ) noch eine tief geordnete Basis-Regel (jede Nominalphrase trägt Nominativ) angenommen wird. Wie Tabelle T15 zeigt, wird die Basis-Regel zwar in den typischen Kasusrektionskontexten durch höher geordnete Regeln außer Kraft gesetzt; aber wenn diese höher geordneten Regeln alle leer erfüllt sind, tritt die tiefstgeordnete Regel in Aktion und sagt korrekt den unmarkierten Fall vorher; vgl. Tabelle T16.

T15: Akk-Regel > Basis-Regel

\begin{tabular}{|l|c|c|c|c|}
\hline I: getroffen, habe, ... & Gen-Regel & Akk-Regel & Nom-Regel & Basis-Regel \\
\hline *01: dass ich ihn getroffen habe & & & & $*$ \\
\hline 02: dass ich er getroffen habe & & $* !$ & & \\
\hline
\end{tabular}

T16: Basis-Regel

\begin{tabular}{|l|c|c|c|c|}
\hline I: und, ... & Gen-Regel & Akk-Regel & Nom-Regel & Basis-Regel \\
\hline 01: Den und ein Buch lesen? & & & & $* !$ \\
\hline - O2: Der und ein Buch lesen? & & & & \\
\hline
\end{tabular}




\section{Schluss}

Ich habe zu zeigen versucht, dass die deutsche Syntax viele Eigenschaften mit der StVO gemein hat, und dass in beiden Fällen die Optimalitätstheorie ein geeignetes Instrument sein kann, richtige (grammatische) von falschen (ungrammatischen) Outputs zu unterscheiden. Es sieht so aus, als sei die Optimalitätstheorie für all solche Regelsysteme relevant, in denen Konzepte wie Regelkonflikt, Reparatur und unmarkierter Fall eine Rolle spielen, und zwar interessanterweise unabhängig davon, ob das Regelsystem wie die StVO per Konvention zustande gekommen oder kognitiv bzw. biologisch verankert ist, und ob es wie bei der Syntax um die menschliche Sprache oder einen anderen Gegenstandsbereich geht. Einschlägige nichtkonventionalisierte, nicht-sprachliche Regelsysteme gibt es im Bereich der Biologie (z.B. die Hierarchie der Instinkttätigkeiten), der Psychologie (z.B. das System der Appetenz- und Aversionskonflikte) und der Informatik (z.B. die Theorie der neuronalen Netze). Innerhalb der Sprachwissenschaft spielt die Optimalitätstheorie eine Rolle zuallererst noch für die Phonologie (für die sie tatsächlich ursprünglich entwickelt worden ist) und die Morphologie. Es gibt aber auch schon Argumente für eine optimalitätstheoretische Semantik, und eine Anwendung des Systems in anderen Bereichen der Sprachwissenschaft scheint zumindest nicht unplausibel.

Naturgemäß sind in dieser Darstellung viele wichtige Aspekte unberïcksichtigt geblieben. Nicht einmal erwähnt habe ich z.B. die weit verbreitete und in der aktuellen Forschung prominente Annahme, dass die grammatischen Regeln im Bereich der Sprache alle universell sind und dass Sprachvariation letztlich immer auf die unterschiedliche Gewichtung dieser invarianten Regeln zurückzuführen ist; Sprachtypologie wird somit per Regelumordnung betrieben. (Eine Übertragung dieser Idee auf die Straßenverkehrsordnung führt partiell zu interessanten Systemen - etwa solchen, bei denen das Absprache-Verbot leichter verletzbar ist, oder solchen, bei denen auch Krankenwagen mit Blaulicht an roten Ampeln halten müssen - , partiell zu weniger interessanten - etwa solchen, bei denen die Anweisungen von Verkehrspolizisten weniger schwer wiegen als Verkehrszeichen.) Ebenso wenig habe ich mich hier mit den vielen neuen Problemen beschäftigt, die die Optimalitätstheorie im Vergleich zu Standardgrammatiken mit sich bringt. Eines davon sei aber doch noch kurz erwähnt: Man kann sich leicht klar machen, dass in einer Grammatik, in der Regeln systematisch verletzbar sind und ein grammatischer Satz somit nicht perfekt, sondern eben nur besser als alle Konkurrenten sein muss, leicht die Situation entsteht, dass man für einen gegebenen Input nicht ohne weiteres einen grammatischen, optimalen Output finden kann. Ein Satz wie *Welches Buch ist Fritz eingeschlafen, nachdem er gelesen hat? ist fraglos ungrammatisch, aber wie lautet dann der grammatische, optimale Satz, der diesen Satz blockiert? Eine mögliche
Antwort könnte wiederum auf den bei den Verkehrsregeln bereits erwähnten leeren Output Ø Bezug nehmen: Bevor man etwas sehr Falsches sagt, ist es vielleicht besser, dass man schweigt.

\section{Anmerkungen}

${ }^{1}$ Vgl. Prince, Alan/ Smolensky, Paul (1993): Optimality Theory. Constraint Interaction in Generative Grammar, Ms., Rutgers University; sowie McCarthy, John/Prince, Alan (1995): Faithfulness and Reduplicative Identity. In: Beckman, Jill/Walsh-Dicki, Laura/ Urbanczyk, Suzanne (Hrsg.): Papers in Optimality Theory. Amherst, Massachussetts: UMass Occasional Papers in Linguistics 18, S. 249-384. Eine gute Einführung auf der Basis der Phonologie ist Kager, René (1999): Optimality Theory. Cambridge: Cambridge University Press; zu einem Überblick über die Syntax vgl. Müller, Gereon (2000): Elemente der optimalitätstheoretischen Syntax. Tübingen: Stauffenburg.

${ }^{2}$ Vgl. Janiszewski, Horst (1998): Straßenverkehrsrecht. München: Deutscher Taschenbuch Verlag \& C.H. Beck; sowie zu einer auf die StVO Bezug nehmenden linguistischen Analyse (wenn auch mit anderem Schwerpunkt) Savigny, Eike (1983): Zum Begriff der Sprache. Stuttgart: Reclam.

${ }^{3}$ Dieser Umstand macht V-Real zu einer sog. translokalen Regel, weil bei der Überprüfung dessen, ob sie von einem gegebenen Output eingehalten wird, Information über andere Outputs wesentlich sein kann.

${ }^{4}$ Wollte man versuchen, die Vorfahrtsregeln so umzuformulieren, dass keine Konflikte entstehen, dann müsste man für jede Vorfahrtsregel sämtliche darüber stehenden Regeln als Ausnahmeklauseln formulieren. Auf diese Weise würde die Information vervielfacht und die Regeln würden in einem Maße komplex, dass sie kaum noch zugänglich wären.

${ }^{5}$ Vgl. u.a. Lenerz, Jürgen (1977): Zur Abfolge nominaler Satzglieder im Deutschen. Tübingen: Stauffenburg; Hoberg, Ursula (1997): Die Linearstruktur des Satzes. In: Grammatik der deutschen Sprache, von Gisela Zifonun, Ludger Hoffmann, Bruno Strecker et al. S. 1495-1680, Berlin: de Gruyter; Choi, Hye-Won (1999): Optimizing Structure in Context, Stanford: CSLI Publications; Büring, Daniel (2001): Let's Phrase It! In: Müller, Gereon/Sternefeld, Wolfgang (Hrsg.): Competition in Syntax. Berlin: Mouton de Gruyter, S. 69-105.

${ }^{6}$ Die hier vorgestellte Analyse geht im Kern zurück auf Schmid, Tanja (2000): Die Ersatzinfinitivkonstruktion im Deutschen. In: Linguistische Berichte 183, S. 325-351.

${ }^{7}$ Vgl. Helbig, Gerhard (1974): Bemerkungen zu den Pronominaladverbien und zur Pronominalität. In: Deutsch als Fremdsprache 11, S. 270-279; Breind1, Eva (1989): Präpositionalobjekte und Präpositionalobjektsätze im Deutschen. Tübingen: Niemeyer.

${ }^{8}$ Diese Regeln sind u.U. phonologisch motivierbar. Vgl. zu einer Verallgemeinerung Müller, Gereon (2000): Das Pronominaladverb als Reparaturphänomen. In: Linguistische Berichte 182, S. 139-178.

Der Autor ist wissenschaftlicher Mitarbeiter am Institut für Deutsche Sprache in Mannheim. 